\title{
Immediate in vivo target-specific cancer cell death after near infrared photoimmunotherapy
}

\author{
Makoto Mitsunaga', Takahito Nakajima', Kohei Sano', Gabriela Kramer-Marek², Peter L Choyke \\ and Hisataka Kobayashi ${ }^{{ }^{*}}$
}

\begin{abstract}
Background: Near infrared (NIR) photoimmunotherapy (PIT) is a new type of cancer treatment based on a monoclonal antibody (mAb)-NIR phthalocyanine dye, (IR700) conjugate. In vitro cancer-specific cell death occurs during NIR light exposure in cells previously incubated with mAb-IR700 conjugates. However, documenting rapid cell death in vivo is more difficult.
\end{abstract}

Methods: A luciferase-transfected breast cancer cell (epidermal growth factor receptor+, MDA-MB-468luc cells) was produced and used for both in vitro and in vivo experiments for monitoring the cell killing effect of PIT. After validation of cytotoxicity with NIR exposure up to $8 \mathrm{~J} / \mathrm{cm}^{2}$ in vitro, we employed an orthotopic breast cancer model of bilateral MDA-MB-468luc tumors in female athymic mice, which subsequently received a panitumumab-IR700 conjugate in vivo. One side was used as a control, while the other was treated with NIR light of dose ranging from 50 to $150 \mathrm{~J} / \mathrm{cm}^{2}$. Bioluminescence imaging (BLI) was performed before and after PIT.

Results: Dose-dependent cell killing and regrowth was successfully monitored by the BLI signal in vitro. Although tumor sizes were unchanged, BLI signals decreased by $>95 \%$ immediately after PIT in vivo when light intensity was high $\left(>100 \mathrm{~J} / \mathrm{cm}^{2}\right)$, however, in mice receiving lower intensity NIR $\left(50 \mathrm{~J} / \mathrm{cm}^{2}\right)$, tumors recurred with gradually increasing BLI signal.

Conclusion: PIT induced massive cell death of targeted tumor cells immediately after exposure of NIR light that was demonstrated with BLI in vivo.

Keywords: Photoimmunotherapy, Theranostics, Cell death, Epidermal growth factor receptor, Molecular targeting, Monoclonal antibody, Bioluminescence imaging

\section{Background}

Conventional cancer therapies cause damage or toxicity in normal tissues, thus requiring dose reductions, which, in turn, limit the effectiveness of such agents $[1,2]$. In general, treatments that maximize target-cell killing while minimizing damage to normal cells are highly desirable. Targeted molecular cancer therapies offer the promise of more effective tumor targeting with fewer side effects than conventional cancer therapies, however, only limited success has thus far, been achieved. Combining drugs with activating physical energy, such as light or heat, is a potential method of

\footnotetext{
* Correspondence: kobayash@mail.nih.gov

${ }^{1}$ Molecular Imaging Program, Center for Cancer Research, National Cancer Institute, National Institutes of Health, Building 10, Room B3B69, MSC1088, Bethesda, MD 20892-1088, USA

Full list of author information is available at the end of the article
}

improving therapeutic selectivity. We recently reported a new type of highly selective cancer therapy, termed "photoimmunotherapy" or PIT, which utilizes a monoclonal antibody (mAb)-bound to the photosensitizing phthalocyanine dye, IRDye700DX (IR700) to target cancer cells and an exposure of near infrared (NIR) light to specifically kill those cells. Remarkably, the mAb-IR700 conjugate is only active as a therapeutic agent, when it is bound to the target cell membrane; otherwise it had no effect on adjacent non-expressing cells [3]. Following NIR light exposure, immediate, target-selective necrotic cell death was observed in vitro using cytotoxicity assays, however, in vivo assessment of rapid cell death before decreasing tumor size is more challenging. Although progressive tumor shrinkage in vivo was observed 3-4 days after PIT, even after only a single administration of mAb-IR700 and

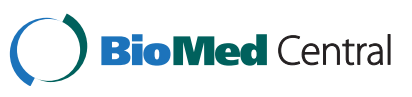


a single exposure of NIR light, nonetheless there is uncertainty over how quickly cell death occurs [4]. Such information could be useful in optimizing PIT dosing and light exposure.

Bioluminescence (BLI) is a well established method of determining in vivo viability [5,6], since the BLI reaction requires both oxygen and ATP to actively transport the substrate luciferin and subsequently catalyze the photochemical reaction [7]. In this study we used BLI to monitor the kinetics of tumor cell death after PIT in epidermal growth factor receptor (EGFR) expressing orthotopic breast tumors after the mouse received anti-EGFR panitumumab-IR700 conjugate (Pan-IR700) followed by varying intensities of NIR light. Results were compared to identical tumors that were not exposed to NIR in the same mice. This method allows for the detection of massive cellular death in vivo immediately after PIT.

\section{Methods}

\section{Reagents}

A water soluble, silicon-phthalocyanine derivative, IRDye 700DX NHS ester (IR700; $\mathrm{C}_{74} \mathrm{H}_{96} \mathrm{~N}_{12} \mathrm{Na}_{4} \mathrm{O}_{27} \mathrm{~S}_{6} \mathrm{Si}_{3}$, molecular weight of 1954.22) was obtained from LI-COR Bioscience (Lincoln, NE). Panitumumab, a fully humanized $\mathrm{IgG}_{2}$ mAb directed against the human EGFR, was purchased from Amgen (Thousand Oaks, CA). All other chemicals were of reagent grade.

\section{Synthesis of IR700-conjugated panitumumab}

Panitumumab (1 mg, $6.8 \mathrm{nmol}$ ) was incubated with IR700 (66.8 $\mu \mathrm{g}, 34.2 \mathrm{nmol}, 5 \mathrm{mmol} / \mathrm{L}$ in DMSO) in 0.1 $\mathrm{mol} / \mathrm{L} \mathrm{Na}_{2} \mathrm{HPO}_{4}(\mathrm{pH} 8.5)$ at room temperature for $2 \mathrm{~h}$. The mixture was purified with a Sephadex G50 column (PD-10; GE Healthcare, Piscataway, NJ). The protein concentration was determined with Coomassie Plus protein assay kit (Thermo Fisher Scientific Inc, Rockford, IL) by measuring the absorption at $595 \mathrm{~nm}$ with spectroscopy (8453 Value System; Agilent Technologies, Santa Clara, CA). The concentration of IR700 was measured by absorption with spectroscopy to confirm the number of fluorophore molecules conjugated to each mAb molecule. The number of IR700 per antibody was $\sim 3$.

\section{Cells}

EGFR-expressing MDA-MB-468luc, stable luciferasetransfected cells [8] were grown in RPMI 1640 supplemented with $10 \%$ fetal bovine serum and $1 \%$ penicillin/ streptomycin in tissue culture flasks in a humidified incubator at $37^{\circ} \mathrm{C}$ in an atmosphere of $95 \%$ air and $5 \%$ carbon dioxide. Balb/3T3 cells (ATCC, Rockville, MD) were used as a control in the same culture condition.

\section{Fluorescence microscopy}

To detect the antigen specific localization of IR700, fluorescence microscopy was performed (BX51 or IX81; Olympus America, Melville, NY). MDA-MB-468luc or 1:1 mixture of MDA-MB-468luc and Balb/3T3 cells were seeded on a cover glass-bottomed dishes and incubated $24 \mathrm{~h}$. Pan-IR700 was added to the culture medium at $10 \mu \mathrm{g} / \mathrm{mL}$ and incubated for $6 \mathrm{~h}$ at $37^{\circ} \mathrm{C}$, then cells were washed with PBS. The filter was set to detect IR700 fluorescence with a 590-650 nm excitation filter, and a 665-740 $\mathrm{nm}$ band pass emission filter.

\section{In vitro $\mathrm{PIT}$}

Cells were seeded into 96 well plate or $35 \mathrm{~mm}$ cell culture dishes and incubated $8 \mathrm{~h}$. Medium was replaced with fresh culture medium containing $10 \mu \mathrm{g} / \mathrm{ml}$ of PanIR700 and incubated over night at $37^{\circ} \mathrm{C}$. After washing with PBS, phenol red free culture medium was added. Then, cells were irradiated with a red light-emitting diode (LED), which emits light at 670 to $710 \mathrm{~nm}$ wavelength (L690-66-60; Marubeni America Co., Santa Clara, $\mathrm{CA})$, and a power density of $25 \mathrm{~mW} / \mathrm{cm}^{2}$ as measured with optical power meter (PM 100, Thorlabs, Newton, NJ).

\section{Phototoxicity assay}

Cytotoxic effects of PIT with Pan-IR700 were determined with luciferase activity assay and flowcytometric LIVEDEAD $^{\oplus}$ Fixable Green Dead Cell Stain Kit (Invitrogen, Carlsbad, CA), which can detect compromised cell membranes. For luciferase activity assay, D-luciferin (Gold Biotechnology, St. Louis, MO) was added to culture media at $150 \mu \mathrm{g} / \mathrm{ml}$ and analyzed on a bioluminescence imaging system (Photon Imager; Biospace Lab, Paris, France). For the flowcytometric assay, cells were trypsinized after treatment and washed with PBS. Green fluorescent reactive dye was added in the cell suspension and incubated at room temperature for 30 min, followed by analysis on a flow cytometer (FACS Calibur, BD Biosciences, San Jose, CA).

\section{Orthotopic breast tumor model}

All in vivo procedures were conducted in compliance with the Guide for the Care and Use of Laboratory Animal Resources (1996), US National Research Council, and approved by the National Cancer Institute Animal Care and Use Committee. Six- to eight-week-old female homozygote athymic nude mice were purchased from Charles River (NCI-Frederick, Frederick, MD). During the procedure, mice were anesthetized with isoflurane. Two million MDA-MB-468luc cells were implanted into the mammary fat pads bilaterally. D-luciferin $(15 \mathrm{mg} / \mathrm{ml}$, $200 \mu \mathrm{l})$ was injected intraperitoneally into mice 6 days after cell implantation, and analyzed with Photon Imager for luciferase activity. Mice were selected for further 
study if their tumors demonstrated symmetry based on size and BLI signal.

\section{In vivo PIT with Pan-IR700}

As there was no treatment effect for MDA-MB-468luc tumors after the single administration of unconjugated panitumumab, selected mice were randomized into 5 groups of 5 animals per group for the following treatments: (1) no treatment; (2) $100 \mu \mathrm{g}$ of Pan-IR700 i.v., no NIR light exposure; (3) $100 \mu \mathrm{g}$ of Pan-IR700 i.v., NIR light was administered at $50 \mathrm{~J} / \mathrm{cm}^{2}$ on day 1 after injection; (4) $100 \mu \mathrm{g}$ of Pan-IR700 i.v., NIR light was administered at $100 \mathrm{~J} / \mathrm{cm}^{2}$ on day 1 after injection; (5) $100 \mu \mathrm{g}$ of Pan-IR700 i.v., NIR light was administered at $150 \mathrm{~J} / \mathrm{cm}^{2}$ on day 1 after injection. NIR light exposure was performed 8 days after cell implantation. Mice images were acquired over time with a fluorescence imager (Pearl Imager; LI-COR Biosciences) for detecting IR700 fluorescence, and Photon Imager for BLI. For analyzing fluorescence and BLI, regions of interest (ROI) of similar size were placed over the entire tumor. The average fluorescence intensity of each ROI was measured. When comparing fluorescence target-to-background ratios (TBR), ROIs were placed in the surrounding non-tumor region.

\section{Histological analysis}

To evaluate serial histological changes immediately after PIT with various NIR light doses, microscopic study was performed (BX51, Olympus America). MDA-MB-468luc tumors were harvested in $10 \%$ formalin immediately after 50,100 , and $150 \mathrm{~J} / \mathrm{cm}^{2}$ of NIR light exposure. Serial $10-\mu \mathrm{m}$ slice sections were fixed on 2 glass slides with Hematoxylin and Eosin (H-E) staining.

\section{Statistical analysis}

Data are expressed as means \pm s.e.m. from a minimum of three experiments. Statistical analyses were carried
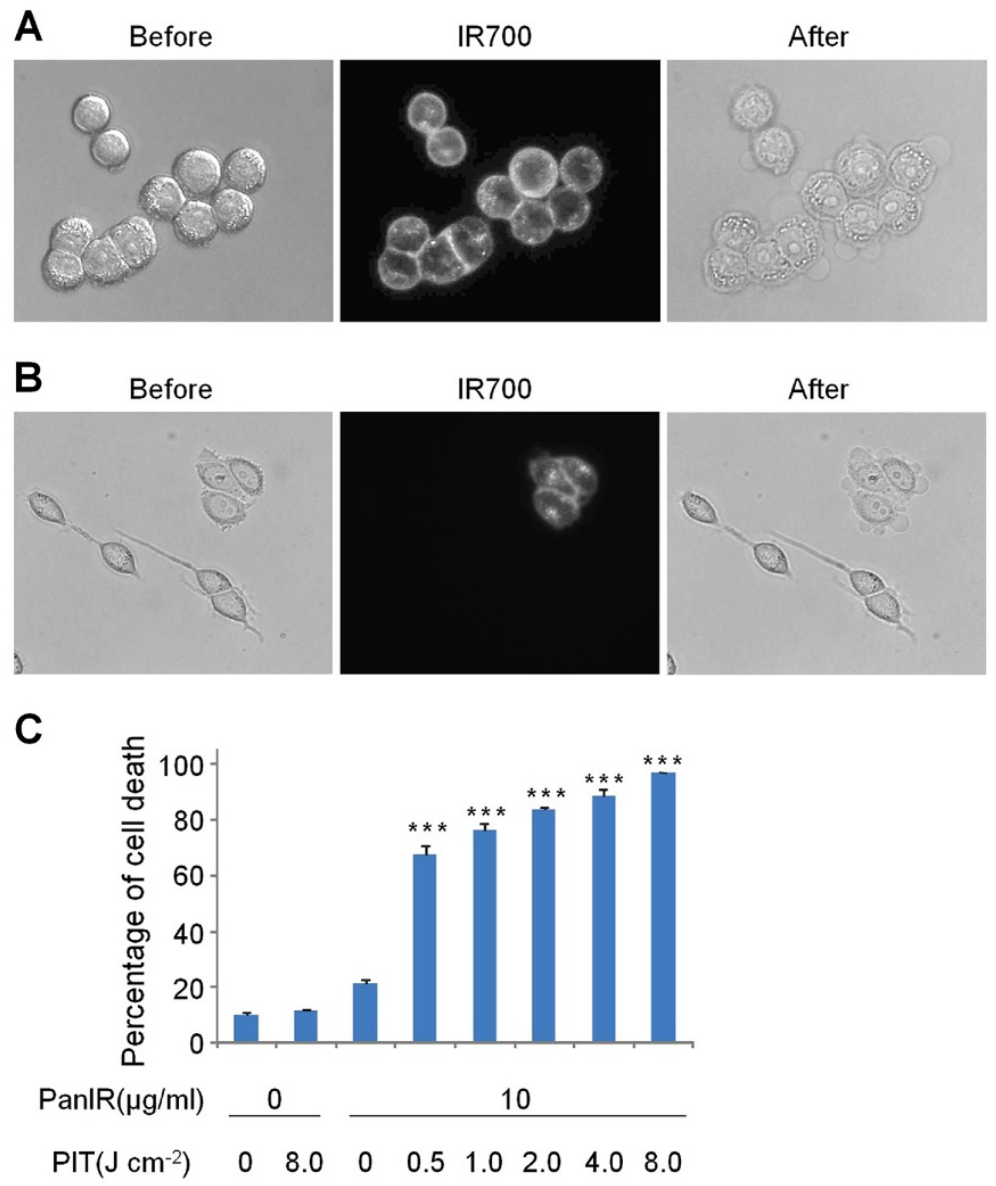

Figure 1 EGFR-specific PIT in vitro. (A) MDA-MB-468luc cells were treated with Pan-IR700 and observed with microscopy (Before and after PanIR700). Necrotic cell death was observed upon excitation with NIR light (After). (B) Both EGFR positive (MDA-MB-468luc) and negative (Balb/3T3) cells were co-cultured and treated with Pan-IR700 (Before and after Pan-IR700). EGFR-specific necrotic cell death was observed (After). (C) NIR light irradiation results in dose dependent cell death as determined by the cytotoxicity assay. $\left(n=3,{ }^{* * *} P<0.001\right.$ vs. non treatment control, Student's $t$ test). 
out using a statistics program (GraphPad Prism; GraphPad Software, La Jolla, CA). Student's $t$ test was used to compare the treatment effects with that of controls.

\section{Results}

Target-specific, dose-dependent NIR light-induced necrotic cell death in response to Pan-IR700 mediated PIT Fluorescence microscopy was performed to confirm targetspecific localization of Pan-IR700. IR700 was mainly localized to the cell membrane and lysosomes of EGFR positive MDA-MB-468luc cells. When these cells were observed during continuous NIR light exposure, almost immediate swelling, budding and rupture of the lysosome was observed leading to irreversible cell death (Figure 1A). MDA-MB-468luc and EGFR negative Balb/3T3 cells were co-incubated to confirm cell-specific killing. Pan-IR700 did not localize to Balb/3T3 cells which were not killed by exposure to NIR (Figure 1B). To confirm that rapid phototoxic cell death had occurred, we used the LIVE/DEAD assay, which can detect early cell membrane damage. Cell death increased significantly in a dose dependent manner with the intensity of NIR light $(P<0.001)$ but no cell death was observed in the absence of Pan-IR700 incubation or in the absence of light (Figure $1 \mathrm{C}$ ).

\section{Bioluminescence imaging of rapid tumor killing in response to Pan-IR700 mediated PIT}

We next examined the effect of rapid Pan-IR700mediated phototoxicity using BLI in vitro. Viable MDAMB-468luc cells ranging from $3.1 \times 10^{4}$ to $1.0 \times 10^{6}$ were detected with BLI prior to NIR exposure (Additional file 1 Figure S1). Low levels of NIR light irradiation dose $\left(\sim 2.0 \mathrm{~J} / \mathrm{cm}^{2}\right)$ resulted in initially decreased BLI followed by recovery of signal as the cells regrew. In contrast, higher levels of NIR light exposure $\left(8.0 \mathrm{~J} / \mathrm{cm}^{2}\right)$ resulted in permanent loss of BLI signal. Interestingly, BLI activity did not immediately disappear after NIR light irradiation even with high levels of exposure (Figure 2A) probably because the budding cells released all the necessary components of the BLI photochemical reaction (luciferase, ATP, oxygen, and luciferin) into the surrounding well permitting the reaction to persist even while the cells were evidently dead based on the cytotoxicity assay, which showed prompt increases in the number of dead cells. Exposure to higher doses of NIR led to the death of more than $90 \%$ of the cells and BLI signal remained reduced over time (Figure $2 \mathrm{~B}$ ).

In vivo monitoring of the acute therapeutic effects of PanIR700 mediated PIT

Orthotopically implanted MDA-MB-468luc breast cancer cells were injected into mammary fat pads bilaterally. Tumors were first recognized at approximately $4 \mathrm{~mm}$ in diameter and 6 days after cell injection. BLI was performed

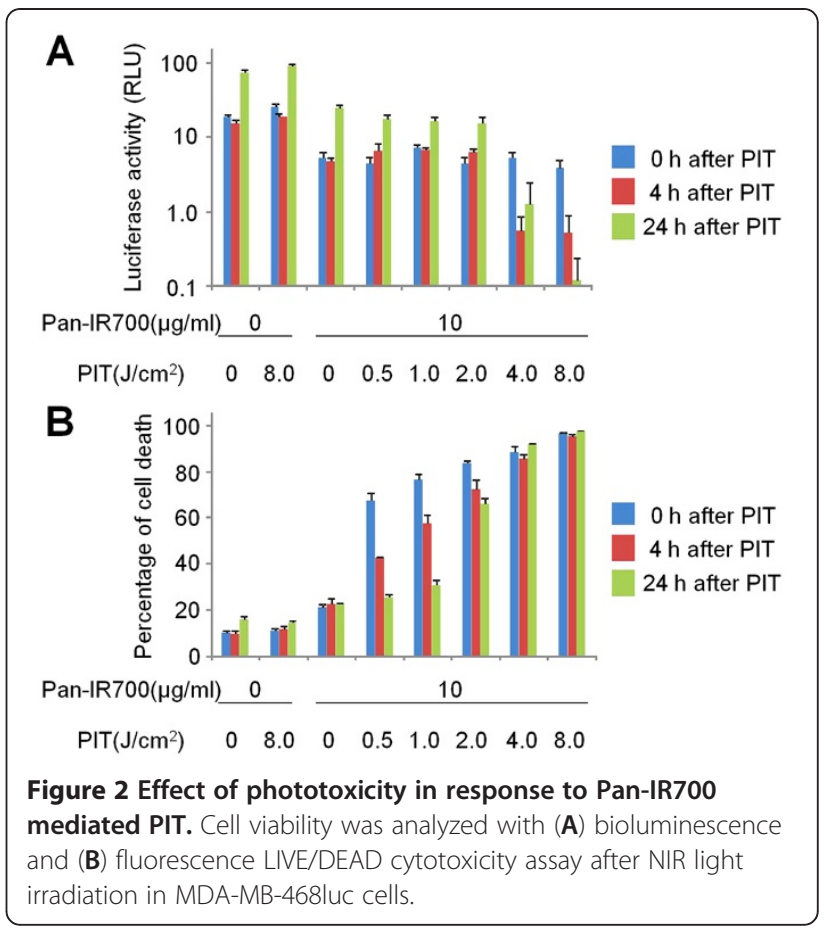

at 6 days to select 25 mice ( 5 groups of 5 mice per group) with symmetrical levels of bioluminescence. In addition to symmetry on BLI we also selected for tumors of similar size and those that demonstrated similar IR700 fluorescence at day 7 post cell injection. Mice received Pan-IR700 at day 6 and typically the right sided tumors were exposed to NIR light irradiation at day 7 , while the left sided tumors were covered. BLI was obtained at $4 \mathrm{~h}$ intervals since this is how long it took for the BLI signal to extinguish after luciferin administration. BLI showed a significant decrease in signal compared to baseline within $4 \mathrm{~h}$ after NIR light exposure, compared with the various control groups: Pan-IR700+ NIR- and Pan-IR700-, NIR + and Pan-IR700-, NIR-. BLI revealed that irradiating with $50 \mathrm{~J} / \mathrm{cm}^{2}$ resulted in the recovery of BLI signal over time, whereas tumors irradiated with $150 \mathrm{~J} / \mathrm{cm}^{2}$ did not show recovery (Figure 3A, B). In order to demonstrate immediate cell killing, we randomized 15 mice in 3 groups $24 \mathrm{~h}$ before NIR irradiation, and irradiated each tumor with $100 \mathrm{~J} / \mathrm{cm}^{2}$, followed by BLI at 0,1 and $4 \mathrm{~h}$ after irradiation. As shown in Figure 3C, BLI signal decreased immediately after NIR light irradiation probably because ATP is hydrolyzed during PIT preventing bioluminescence reactions. Since IR700 is also a fluorescent dye, we were also able to observe PIT effects with a fluorescence camera. IR700 fluorescence was detected in MDA-MB-468luc tumors before NIR light irradiation with a high target-to-background ratio $(\mathrm{TBR}=\sim 8)$, but was markedly decreased to nearly background level ( $\mathrm{TBR}=\sim 1)$ immediately after NIR light irradiation. In contrast, IR700 fluorescence intensity in non-treated 


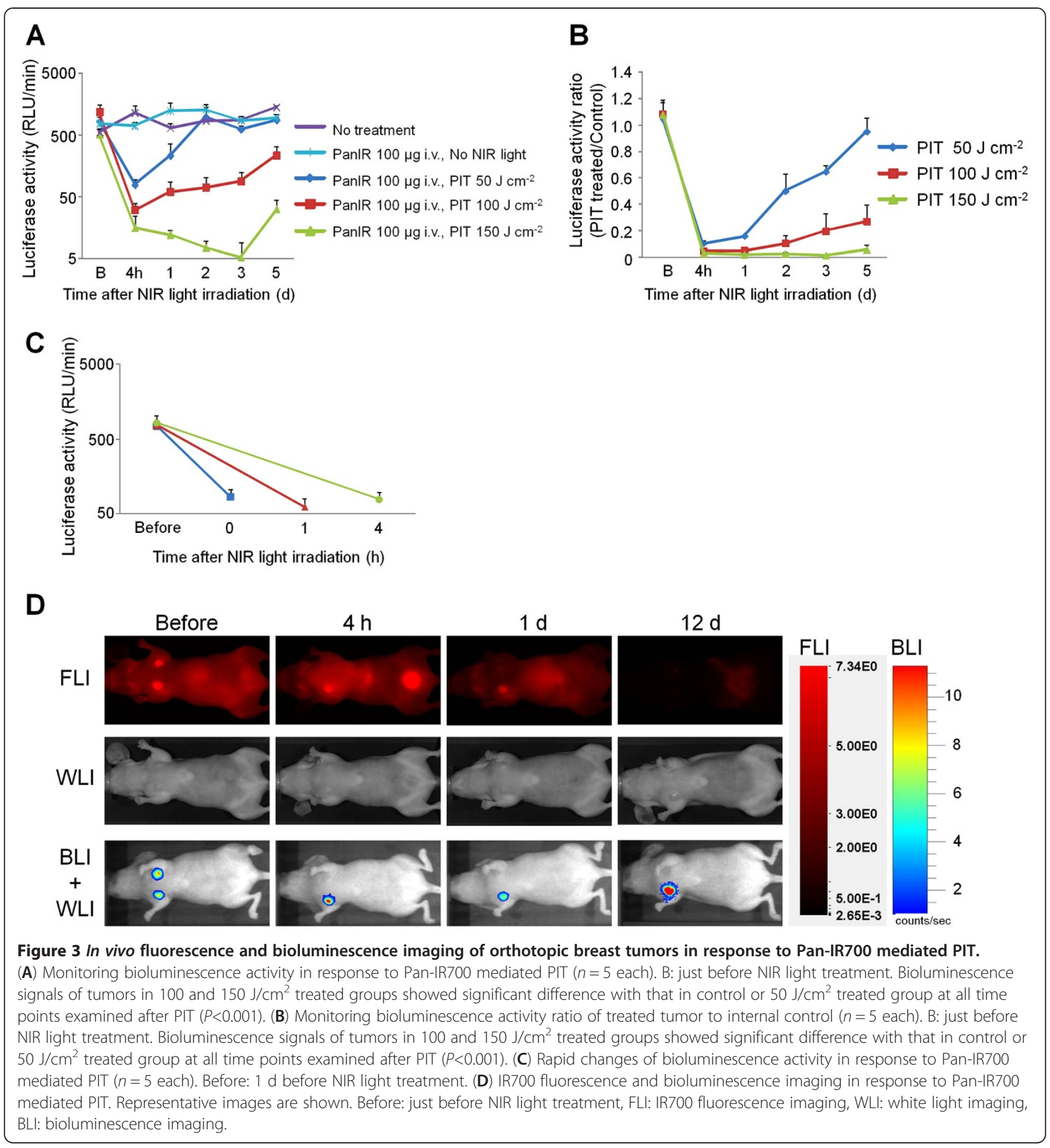

(Pan-IR700+, NIR-) control tumors decreased slowly with time commensurate with the conjugate's pharmacokinetics (Figure 3D).

\section{Histological analysis}

Microscopic evaluation of treated tumors revealed diffuse necrosis and microhemorrhage with scattered clusters of damaged tumor cells after PIT with $50-150 \mathrm{~J} / \mathrm{cm}^{2}$. Necrotic damage was more intense and fewer tumor cells remained, when higher energy $\left(150 \mathrm{~J} / \mathrm{cm}^{2}\right)$ NIR light was administered (Figure 4).

\section{Discussion}

This study demonstrates that target-selective accumulation of Pan-IR700 in MDA-MB-468luc tumors resulted in rapid cell death, which was dose dependent based on the NIR light intensity in the range of $50-150 \mathrm{~J} / \mathrm{cm}^{2}$. Immediate cell death after exposure to NIR light could be 


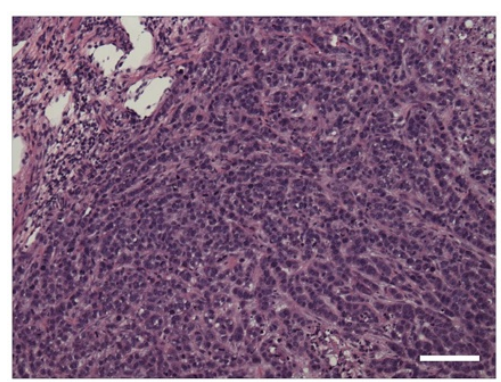

$0 \mathrm{~J} / \mathrm{cm}^{2}$

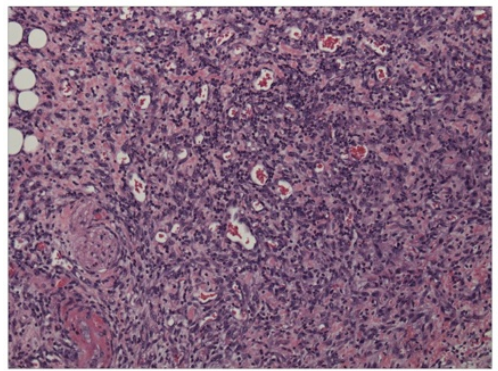

$100 \mathrm{~J} / \mathrm{cm}^{2}$

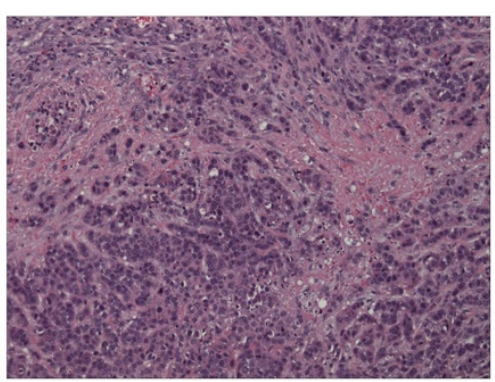

$50 \mathrm{~J} / \mathrm{cm}^{2}$

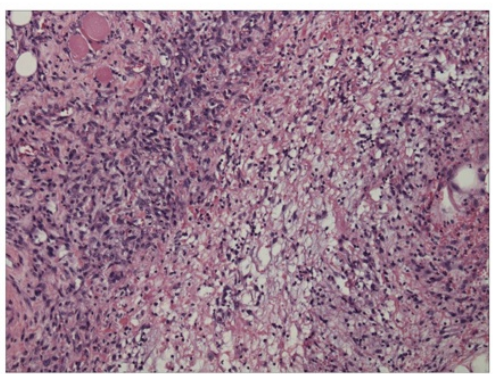

$150 \mathrm{~J} / \mathrm{cm}^{2}$

Figure 4 Histological findings immediately after PIT with various NIR exposures. Histological specimens of MDA-MB-468luc tumors, which were treated with PIT at 0,50,100, and $150 \mathrm{~J} / \mathrm{cm}^{2}$, are shown. All specimens are stained with Hematoxylin and Eosin. A few scattered clusters of damaged tumor cells are seen within a background of diffuse cellular necrosis and micro-hemorrhage immediately after PIT with $50 \mathrm{~J} / \mathrm{cm}^{2}$. Necrotic damage was more intense when higher intensity NIR light was administered. Scale indicates 50 $\mu m$.

validated by BLI. Bioluminescence signals decreased to less than $3 \%$ just after PIT treatment with $100 \mathrm{~J} / \mathrm{cm}^{2}$ of NIR light irradiation, indicating near instantaneous tumor cell killing in vivo. Fluorescence signal also decreased immediately after NIR exposure of PIT as shown in Figure 3D. However, this immediate decrease of IR700 fluorescence could be induced partly by photobleaching of IR700. This immediate cell death suggests that the mechanism of PIT-induced tumor cell death is necrosis via direct physical injury such as pressure waves induced by local heat elevation and not through slower death pathways such as apoptosis or autophagy. Lower doses of light resulted in incomplete cell killing causing tumor regrowth as demonstrated by increasing BLI signal. Higher doses of light (e.g. $150 \mathrm{~J} / \mathrm{cm}^{2}$ ) resulted in complete responses. Thus, BLI are able to monitor therapeutic responses to PIT.

Paradoxically, the BLI results appeared to be less rapid when cells were tested in vitro. Although Pan-IR700 treated MDA-MB-468luc cells were rapidly and selectively killed in response to NIR light irradiation, BLI appeared to show that cell killing was slower than in vivo cell killing [3]. Nearly instantaneous cell killing was demonstrated with the LIVE/DEAD cytotoxicity assay, which detected early cellular membrane damage after low levels (less than $2 \mathrm{~J} / \mathrm{cm}^{2}$ ) of NIR light while BLI signal was reduced only after $4 \mathrm{~h}$ post NIR exposure $[9,10]$. These data suggest that disrupted cellular membranes, which can be defined as "dead" by LIVE/DEAD staining assay may undergo rapid cell surface repair to reseal cellular membrane, while, aggressively disrupted cells after strong NIR irradiation (more than $2 \mathrm{~J} / \mathrm{cm}^{2}$ ) were irreversibly damaged and could not repair the disrupted membrane [11]. However, an additional factor is that BLI signal was artifactually preserved in vitro. Even after severe mechanical disruption of a cell membrane all the necessary elements for BLI including ATP, oxygen and luciferin still exist within the well in sufficient concentrations to produce a photochemical reaction. In contrast, when PIT is performed in vivo, released ATP is rapidly hydrolyzed in the local microenvironment resulting in rapid loss of BLI signal. Thus, BLI may be a more valuable tool for in vivo monitoring than for in vitro monitoring of cell therapies, which are based on rapid physical damage, as opposed to chemical or biological damage to cancer cells.

Fluorescent proteins (FPs) are a potential alternative for monitoring tumor growth in vivo [12-15]. Fluorescence imaging using FPs are better direct and stable method for longitudinal monitoring therapeutic effects of photo-therapy $[16,17]$ for days or weeks than the bioluminescence imaging, which is used in this study, because most of FPs are stable in solution for days in vitro [18] and fluoresced before FPs are taken up and catabolized by macrophages in vivo [12]. Therefore, fluorescence imaging has already been used for longitudinal monitoring of therapeutic effects of PIT [4]. However, 
PIT-induced immediate massive cell death, which rarely happens in cancer therapy, did not depict after with the fluorescence imaging for hours but depicted with the bioluminescence imaging because fluorescent substances are stable than ATP, which hydrized immediately in vivo. Therefore, the bioluminescence imaging is theoretically and practically the appropriate method for detecting this unique PIT-induced immediate massive cell death.

Proper controls are vital to prove that the cell killing is related to the combination of Pan-IR700 and NIR light exposure. We achieved this by implanting breast tumors bilaterally in the fat pads of mice and selecting for mice with tumors that were symmetric in size and BLI/fluorescence signals just before PIT. Controls included tumors that did not receive Pan-IR700 but did receive light, tumors that received Pan-IR700 but did not receive NIR light and those that received neither agent nor light. No cell killing was observed in these controls. In contrast to a previous study of PIT, which employed a subcutaneous xenograft, we employed an orthotopic bilateral breast cancer tumor model and used one tumor as an internal control [19]. Such symmetry is more easily achieved in orthotopic vs. subcutaneous models and this model was also able to demonstrate that response was dose dependent with regard to light exposure.

\section{Conclusions}

Immediate cytotoxicity induced by PIT was demonstrated using bioluminescence imaging in vivo. The immediate cell killing demonstrated by BLI strongly suggests that the mechanism of action of PIT is necrosis due to rapid mechanical membrane disruption caused by local heating and induced pressure waves. This is supported by direct observational microscopic evidence that rapid cell swelling and budding is seen in cells previously treated with a mAb-IR700 conjugate and subsequently exposed to NIR light. This data support the concept that PIT could be highly controlled by appropriate dosing of light to specific tumor cells identified by their IR700 fluorescence, resulting in a true "see and treat" paradigm that could be useful during surgical or endoscopic procedures. Practically, surgeons or endoscopy physicians could "see" tumors with the fluorescence of IR700, and then "treat" them by surgery combined with exposing the NIR light to achieve complete treatment of a patient. This result suggests that physicians should not misread remaining persistent fluorescence signal as a sign of survived tumors.

\section{Additional file}

Additional file 1: Figure S1. Sensitivity of bioluminescence assay in serially diluted viable MDA-MB-468luc cells. BLI signal could be detected in as few as $3.1 \times 10^{4}$ cells MDA-MB-468luc.

\section{Abbreviations}

NIR: Near infrared; PIT: Photoimmunotherapy; mAb: Monoclonal antibody; EGFR: Epidermal growth factor receptor; BLI: Bioluminescence imaging; Pan: Panitumumab; IR700: IRDye700DX; ROI: Regions of interest; TBR:

Target-to-background ratios.

\section{Competing interests}

The authors declare that they have no competing interests.

\section{Authors' contributions}

M.M. conducted experiments, performed analysis and wrote the manuscript; T.N., K.S. and G.K. conducted experiments and performed analysis; P.L.C. wrote the manuscript and supervised the project; and H.K. planned and initiated the project, designed and conducted experiments, wrote the manuscript, and supervised the entire project. All authors read and approved the final manuscript

\section{Acknowledgments}

This research was supported by the Intramural Research Program of the National Institutes of Health, National Cancer Institute, Center for Cancer Research.

\section{Author details}

${ }^{1}$ Molecular Imaging Program, Center for Cancer Research, National Cancer Institute, National Institutes of Health, Building 10, Room B3B69, MSC1088 Bethesda, MD 20892-1088, USA. 2Radiation Oncology Branch, Center for Cancer Research, National Cancer Institute, National Institutes of Health, Bethesda, MD 20892, USA.

Received: 22 May 2012 Accepted: 31 July 2012

Published: 8 August 2012

\section{References}

1. Waldmann TA: Immunotherapy: past, present and future. Nat Med 2003, 9:269-277.

2. Pastan I, Hassan R, Fitzgerald DJ, Kreitman RJ: Immunotoxin therapy of cancer. Nat Rev Cancer 2006, 6:559-565.

3. Mitsunaga M, Ogawa M, Kosaka N, Rosenblum LT, Choyke PL, Kobayashi H: Cancer cell-selective in vivo near infrared photoimmunotherapy targeting specific membrane molecules. Nat Med 2011, 17:1685-1691.

4. Mitsunaga M, Nakajima T, Sano K, Choyke PL, Kobayashi H: Near-infrared theranostic photoimmunotherapy (PIT): repeated exposure of light enhances the effect of immunoconjugate. Bioconjug Chem 2012, 23:604-609.

5. Contag PR, Olomu IN, Stevenson DK, Contag CH: Bioluminescent indicators in living mammals. Nat Med 1998, 4:245-247.

6. Rehemtulla A, Stegman LD, Cardozo SJ, Gupta S, Hall DE, Contag CH, Ross BD: Rapid and quantitative assessment of cancer treatment response using in vivo bioluminescence imaging. Neoplasia 2000, 2:491-495.

7. Dothager RS, Flentie K, Moss B, Pan MH, Kesarwala A, Piwnica-Worms D: Advances in bioluminescence imaging of live animal models. Curr Opin Biotechnol 2009, 20:45-53.

8. Lyakhov I, Zielinski R, Kuban M, Kramer-Marek G, Fisher R, Chertov O, Bindu L, Capala J: HER2- and EGFR-specific affiprobes: novel recombinant optical probes for cell imaging. ChemBioChem 2010, 11:345-350.

9. Leist $M$, Single B, Castoldi AF, Kuhnle S, Nicotera P: Intracellular adenosine triphosphate (ATP) concentration: a switch in the decision between apoptosis and necrosis. J Exp Med 1997, 185:1481-1486.

10. Zamaraeva MV, Sabirov RZ, Maeno E, Ando-Akatsuka Y, Bessonova SV, Okada Y: Cells die with increased cytosolic ATP during apoptosis: a bioluminescence study with intracellular luciferase. Cell Death Differ 2005, 12:1390-1397.

11. McNeil PL, Steinhardt RA: Plasma membrane disruption: repair, prevention, adaptation. Annu Rev Cell Dev Biol 2003, 19:697-731.

12. Hoffman RM: The multiple uses of fluorescent proteins to visualize cancer in vivo. Nat Rev Cancer 2005, 5:796-806.

13. Hoffman RM, Yang M: Subcellular imaging in the live mouse. Nat Protoc 2006, 1:775-782

14. Jiang P, Yamauchi K, Yang M, Tsuji K, Xu M, Maitra A, Bouvet M, Hoffman RM: Tumor cells genetically labeled with GFP in the nucleus and RFP in the cytoplasm for imaging cellular dynamics. Cell Cycle 2006, 5:1 198-1201. 
15. Yamamoto N, Jiang $P$, Yang M, Xu M, Yamauchi K, Tsuchiya H, Tomita K, Wahl GM, Moossa AR, Hoffman RM: Cellular dynamics visualized in live cells in vitro and in vivo by differential dual-color nuclear-cytoplasmic fluorescent-protein expression. Cancer Res 2004, 64:4251-4256.

16. Kimura H, Lee C, Hayashi K, Yamauchi K, Yamamoto N, Tsuchiya H, Tomita K, Bouvet M, Hoffman RM: UV light killing efficacy of fluorescent proteinexpressing cancer cells in vitro and in vivo. J Cell Biochem 2010, 110:1439-1446.

17. Tsai MH, Aki R, Amoh Y, Hoffman RM, Katsuoka K, Kimura H, Lee C, Chang CH: GFP-fluorescence-guided UVC irradiation inhibits melanoma growth and angiogenesis in nude mice. Anticancer Res 2010, 30:3291-3294.

18. Shimomura O: Discovery of green fluorescent protein (GFP) (Nobel Lecture). Angew Chem Int Ed Engl 2009, 48:5590-5602.

19. Hoffman RM: Orthotopic metastatic mouse models for anticancer drug discovery and evaluation: a bridge to the clinic. Invest New Drugs 1999, 17:343-359.

doi:10.1186/1471-2407-12-345

Cite this article as: Mitsunaga et al.: Immediate in vivo target-specific cancer cell death after near infrared photoimmunotherapy. BMC Cancer 2012 12:345

\section{Submit your next manuscript to BioMed Central and take full advantage of:}

- Convenient online submission

- Thorough peer review

- No space constraints or color figure charges

- Immediate publication on acceptance

- Inclusion in PubMed, CAS, Scopus and Google Scholar

- Research which is freely available for redistribution 